\title{
Making context memories independent of the hippocampus
}

\author{
Hugo Lehmann, ${ }^{1,3}$ Fraser T. Sparks, ${ }^{2}$ Simon C. Spanswick, ${ }^{2}$ Crystal Hadikin, ${ }^{2}$ \\ Robert J. McDonald, ${ }^{2}$ and Robert J. Sutherland ${ }^{2}$ \\ ${ }^{7}$ Psychology Department, Trent University, Peterborough, Ontario K9J 7B8, Canada; ${ }^{2}$ Canadian Centre for Behavioural \\ Neuroscience, Department of Neuroscience, The University of Lethbridge, Lethbridge, Alberta T1K 3M4, Canada
}

\begin{abstract}
We present evidence that certain learning parameters can make a memory, even a very recent one, become independent of the hippocampus. We confirm earlier findings that damage to the hippocampus causes severe retrograde amnesia for context memories, but we show that repeated learning sessions create a context memory that is not vulnerable to the damage. The findings demonstrate that memories normally dependent on the hippocampus are incrementally strengthened in other memory networks with additional learning. The latter provides a new account for patterns of hippocampal retrograde amnesia and how memories may become independent of the hippocampus.
\end{abstract}

[Supplemental material is available online at www.learnmem.org.]

Contextual fear conditioning can be supported by two neural systems, one that contains the hippocampus (HPC), and one that does not. Evidence for this assertion comes from studies in which the HPC, in rats, is damaged either before or after the contextual fear conditioning. Extensive damage to the HPC before conditioning has little effect on contextual fear conditioning (Maren et al. 1997; Frankland et al. 1998; Wiltgen et al. 2006). This result can only mean that there is a non-HPC memory system that can support fear of context. In contrast, there is unequivocal evidence that moderate to extensive damage to the HPC soon after learning severely impairs the ability of the conditioning context to evoke fear, suggesting that the HPC normally makes a major contribution to this type of memory (Kim and Fanselow 1992; Maren et al. 1997; Frankland et al. 1998; Anagnostaras et al. 1999; Debiec et al. 2002; Lehmann et al. 2007b; Sutherland et al. 2008; Wang et al. 2009).

The dissociable effects of pre- and post-training HPC damage on contextual fear conditioning have been interpreted as suggesting that: (1) When the HPC is intact during learning it interferes with other systems and prevents them from acquiring an independent contextual fear conditioning memory, and (2) when the HPC is absent, these other systems are released from this interference and are able to rapidly acquire an independent memory (Maren et al. 1997; Frankland et al. 1998; Fanselow and Poulos 2004; Driscoll et al. 2005; Lehmann et al. 2006; Sutherland et al. 2006). The latter interference from the HPC on the other memory systems has been termed overshadowing. Supplemental Figure S1 depicts data from our laboratory demonstrating the overshadowing phenomenon and the dissociable effects of HPC damage induced before and after contextual fear conditioning.

Very little, however, is known about the parameters determining the extent to which the HPC system interferes with the non-HPC system for control over contextual fear. The purpose of the current study is to provide some insight into this issue. Typically, contextual fear conditioning in rats is conducted in a single conditioning session in which a configuration of static

${ }^{3}$ Corresponding author.

E-mail hugolehmann@trentu.ca; fax (705) 748-1580.

Article is online at http://www.learnmem.org/cgi/doi/10.1101//m.1385409. background cues is paired with several footshocks. When returned to the conditioning context, rats display several speciesspecific defensive responses including freezing (i.e., absence of movement except for breathing). Several theorists have proposed that non-HPC systems are more likely to be recruited when there are multiple experiences with similar events, which, in turn, would mitigate the necessity of the HPC for memory expression (O'Keefe and Nadel 1978; Sherry and Schacter 1987; McClelland et al. 1995; O'Reilly and Rudy 2001; White and McDonald 2002). Accordingly, we hypothesized that repeated contextual fear conditioning sessions separated by hours and days would overcome the HPC interference or overshadowing effect. In other words, with repeated learning sessions, enough information would be incrementally captured by the non-HPC system to support a contextual fear memory that would survive complete damage to the HPC.

Adult male rats received 11 fear-conditioning sessions across $6 \mathrm{~d}$. In each session, they were placed in a context and received mild footshocks (Shock Context). Concurrently, the rats were exposed 10 times to another context in which they never received shock (No-Shock Context). The No-Shock Context served as a control condition to measure whether the rats simply showed generalized fear or could show context-specific memory. Within $72 \mathrm{~h}$ following the last conditioning session, rats either received sham surgery or complete lesions of the HPC using the neurotoxin $\mathrm{N}$-methyl-D-aspartic acid (NMDA) (Lehmann et al. 2007a). Rats were then tested for retention in both the Shock and No-Shock Contexts in a counterbalanced order. In addition, in a single learning episode, another group of rats received a matching number of shocks (i.e., 12 shocks) and context exposure (i.e., $17 \mathrm{~min}$ ), and then received surgery 7-10 d after conditioning. The latter interval is identical to the interval between the initial conditioning session and surgery in the repeated learning condition. Figure 1 illustrates and describes the design of the experiments.

When all shocks were delivered in a single session, HPC damage caused profound retrograde amnesia. As illustrated in Figure $2 \mathrm{~A}$, the HPC rats displayed significantly less freezing than control rats during the retention test $\left(t_{(8)}=23.895, P<0.001\right)$. This result replicates all previous studies in which the HPC was damaged days after a single contextual fear conditioning training 
A)

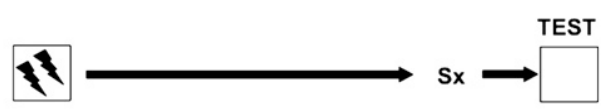

B)

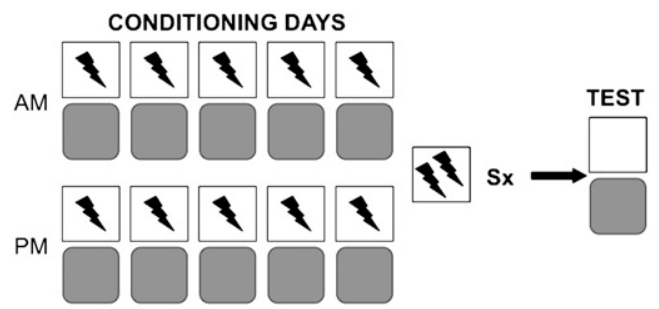

Figure 1. Illustration of the experimental design used in $(A)$ the single conditioning session and $(B)$ repeated conditioning session experiments. In $A$ the rats were initially placed in the conditioning chamber for $17 \mathrm{~min}$ and received the first of 12 footshocks $(1 \mathrm{~mA} / 2 \mathrm{sec})$ at the 300 -sec mark, and then one every following $58 \mathrm{sec}$ after shock offset. Seven to $10 \mathrm{~d}$ later, the rats either received sham or HPC damage (Sx). Approximately $10 \mathrm{~d}$ after, the rats were returned to the chamber to assess freezing over a 5 -min retention test. In $B$ the rats were placed initially in the conditioning chamber for $1 \mathrm{~min}$ and received a shock at the $45-\mathrm{sec}$ mark (Shock Context). Approximately 45 min later, the rats were placed in a different chamber for $1 \mathrm{~min}$ and did not receive shock (No-Shock Context). The procedure was repeated twice daily for five consecutive days, and the Shock and No-Shock chamber order was counterbalanced according to the principles of a Latin Square design. The rats then received sham or HPC damage 1-3 d later. The rats' retention was assessed in both contexts $\sim 10 \mathrm{~d}$ after surgery in both the Shock and NoShock Context in a counterbalanced order with a 24-h span between tests. Importantly, the number of shocks, context exposure time, and interval between initial learning and surgery were matched between both experiments.

session (Kim and Fanselow 1992; Maren et al. 1997; Frankland et al. 1998; Anagnostaras et al. 1999; Debiec et al. 2002; Lehmann et al. 2007b; Sutherland et al. 2008).

In striking contrast, memory for contextual fear conditioning was spared when the HPC was damaged after repeated conditioning sessions. Figure $2 \mathrm{~B}$ shows the percent time spent freezing during the retention test in the Shock and No-Shock Contexts. An ANOVA with between-group factor (Lesion: Sham and HPC) and within-group factor (Context: Shock and No-Shock) revealed a significant main effect of Context $\left(F_{(1,14)}=84.731, P<0.001\right)$, indicating that the rats displayed higher levels of freezing in the Shock than in the NoShock Context. The effect of Lesion $\left(F_{(1,14)}=4.280, P=0.058\right)$ was not significant, nor was the Lesion $\times$ Context interaction $\left(F_{(1,14)}=0.877, P=0.369\right)$, suggesting that extensive HPC damage did not impair memory. The tendency for an effect of Lesion is due to the HPC rats freezing less than the Sham rats in the No-Shock Context $(P=0.06)$ rather than freezing less in the Shock Context $(P=$ 0.457).

The repeated conditioning sessions clearly enabled a contextual fear representation to be established in non-HPC memory systems. However, it is surprising that the HPC damage did not impair the ability to discriminate between the Shock and No-Shock Context, because evidence suggests that context discrimination is dependent on the HPC (see Moscovitch et al. 2006). Indeed, studies of rats with HPC damage induced before learning have shown that contextual fear conditioning is acquired quickly by non-HPC systems in a single session, but the ability to discriminate between the training context and a new context is lost (Frankland et al. 1998; Antoniadis and McDonald 2000; Winocur et al. 2007). Hence, it is significant in the present study that the HPC damage did not impair context discrimination abilities in the rats that received repeated learning episodes. The latter appear to have established a context representation, outside of the HPC, that was not bereft of details. Yet, one should consider that the rats in the repeated sessions experiment received experience in both the Shock and No-Shock Contexts prior to surgery, and this discrimination training procedure may have established two different non-HPC representations. It remains possible that HPC damage would impair the ability to discriminate the Shock Context from a new context, which is what is found in anterograde amnesia studies (Frankland et al. 1998; Antoniadis and McDonald 2000; Winocur et al. 2007). To address this possibility, a new experiment examined whether HPC-damaged rats could discriminate the Shock Context from a Novel Context. Rats were trained with the same repeated learning protocol as described earlier, with the exception that the rats were never placed in the No-Shock Context prior to surgery. One to $3 \mathrm{~d}$ following learning, the rats either received Sham or complete HPC damage. They were then tested for retention in the Shock and the Novel (i.e., No-Shock) Context in a counterbalanced order. Figure 3 shows the percent time spent freezing during the retention test in the Shock and Novel Contexts. An ANOVA with between-group factor (Lesion: Sham and HPC) and within-group factor (Context: Shock and Novel) revealed that the rats froze significantly more in the Shock than the Novel Context $\left(F_{(1,10)}=57.393, P<0.001\right)$. However, no significant difference was found between the HPC and Sham groups $\left(F_{(1,10)}=0.597, P=0.458\right)$ and the Lesion $\times$ Context interaction did not reach significance $\left(F_{(1,10)}=0.123, P=0.733\right)$. Thus, as in the previous repeated sessions experiment, the HPC damage did not cause retrograde amnesia for contextual fear conditioning and, more importantly, the HPC damage did not impair the ability to discriminate between the original context and new context.
A)

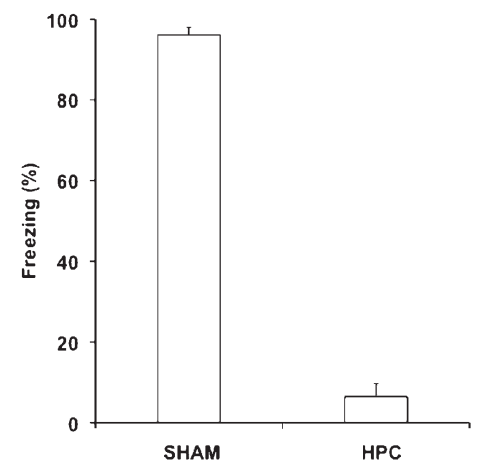

B)

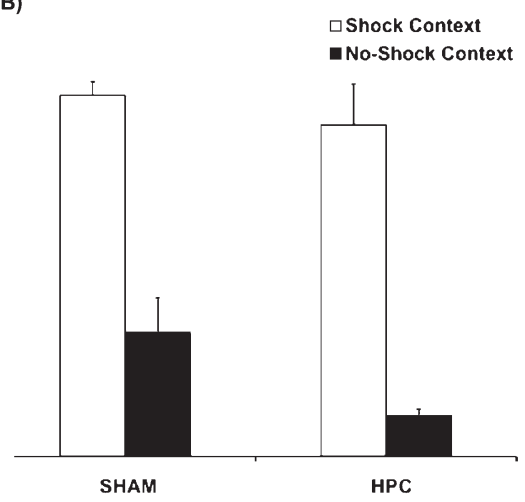

Figure 2. Mean ( \pm SEM) percent time freezing by Sham and HPC rats during the retention test of the (A) single conditioning (12 shocks) experiment and (B) repeated conditioning session experiment. In $A$ the HPC rats showed significantly less freezing $(P<0.001)$ than the Sham rats, suggesting that the damage caused profound retrograde amnesia for contextual fear conditioning learned in a single session 7-10 d before surgery. In $B$ the performance of the HPC rats did not significantly differ from the Sham rats, and they exhibited significantly more freezing in the Shock Context than the No-Shock Context $(P<0.001)$. Consequently, repeated conditioning sessions prevented the retrograde amnesic effects normally observed in contextual fear conditioning following HPC damage, suggesting that other neural networks were now able to support the memory. 


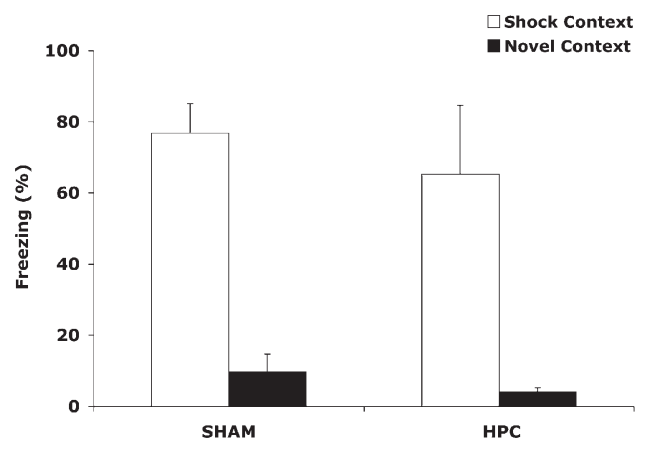

Figure 3. Mean ( \pm SEM) percent time freezing by Sham and HPC rats in the Shock and Novel Contexts during the retention tests of the discrimination experiment. The rats exhibited significantly more freezing in the Shock than the Novel Context $(P<0.001)$, and the HPC rats did not significantly differ from the Sham rats, suggesting that the HPC-damaged rats remembered the specific meaning of the Shock Context as well as control rats. Hence, repeated conditioning sessions established a contextrich representation in non-HPC systems, which supports successful context discriminations.

The absence of amnesia for contextual fear conditioning in the current study is not due to insufficient damage to the HPC. We calculated (see Lehmann et al. 2007b) that an average of $83 \%$ of the HPC was damaged across rats (smallest: 64\%; largest: 90\%) in the repeated learning experiments (see Supplemental material for more histological details). The amount of HPC damage is substantially more than that found in most studies reporting impairments for contextual fear conditioning following HPC damage (Kim and Fanselow 1992; Maren et al. 1997; Frankland et al. 1998; Anagnostaras et al. 1999; Debiec et al. 2002) and more than for the single-session experiment (average 76\%) in which we currently report amnesia. Therefore, the amount of HPC damage inflicted in the rats in this study is certainly sufficient to disrupt HPC-dependent memories.

Like others (Kim and Fanselow 1992; Maren et al. 1997; Frankland et al. 1998; Anagnostaras et al. 1999; Debiec et al. 2002; Lehmann et al. 2007b; Sutherland et al. 2008), we found that damage to the HPC after a single contextual fear-conditioning session involving multiple shocks produces profound retrograde amnesia for contextual fear conditioning. However, in two separate experiments, distributing shock across multiple conditioning sessions prevented this amnesia. In one case, the rats experienced Context-Shock pairings in one context and no shock in another context. Following this training, rats with damage to the HPC did not differ from control rats in the absolute amount of freezing in the training context nor in their ability to discriminate between the two contexts. In the second case, rats only received the multiple Context-Shock sessions. Rats with damage to the HPC could not be distinguished from control rats during the test in the training context or in their responses to a novel context. These findings provide new support for the general idea that contextual fear conditioning can be supported by both HPC and non-HPC systems. This conclusion is supported by (1) the finding that damage to the HPC following a single conditioning session virtually eliminates freezing during the test, implying the importance of the HPC system, and (2) that following multiple conditioning sessions, damage to the HPC has no effect on either contextual fear displayed in the training context or their ability to discriminate the training context from other contexts, suggesting the existence of non-HPC systems that can support contextual fear. The findings also reveal that the overshadowing or interference by the HPC over the non-HPC memory systems for control over contextual fear is not absolute. Following a single conditioning session, removal of the HPC produced a devastating retrograde amnesia, illustrating substantial overshadowing. However, distributing conditioning across several sessions completely attenuated the effects of damage to the HPC, revealing that non-HPC systems can support contextual fear conditioning despite the HPC, and revealed the importance of multiple sessions for this to occur.

The overshadowing by the HPC is based on the familiar idea in associative learning at the behavioral level, where through a competitive process some of the cues that redundantly predict a reinforcer acquire the ability to generate strong conditioned responding, while other equally predictive, but less salient cues do not (Stout et al. 2003). Conditioning to the less potent cues proceeds more effectively if the more potent competitors are absent. Following the same principle, if the HPC representation is active, then learning in the non-HPC systems suffers strong interference. In contrast, in the absence of the HPC representation, learning in non-HPC systems is released from this interfering effect of the HPC. Thus, the learning rate in non-HPC networks is potently lowered by the activity of the HPC. However, with repeated learning, other structures, which are overshadowed by the HPC, may cumulatively build a representation that achieves HPC independence. The current findings clearly support this hypothesis, whereby repeated learning episodes incrementally established a contextual fear-conditioning representation outside of the HPC that mitigated the usual retrograde amnesic effects of HPC damage.

One important question is where does the HPC interference occur? Biedenkapp and Rudy (2009) recently reported that the HPC competes with the basolateral region of the amygdala during fear conditioning. Previously, Guarraci et al. (1999) found that the amount of conditioned fear produced by training could be increased if the dopamine D1 receptor agonist SKF82958 was injected into the basolateral region. Biedenkapp and Rudy (2009) reasoned that if this is the area where the HPC interferes with nonHPC systems for the association with shock, then a local infusion of SKF82958 before a single session of contextual fear conditioning should attenuate the interference and allow the non-HPC system to gain more control over contextual fear. Their data supported this hypothesis, which leads to the possibility that with multiple conditioning sessions, the non-HPC system gradually gains association with these fear-supporting neurons in this region of the brain.

Patients with bilateral damage to the HPC often exhibit temporally graded retrograde amnesia, such that recently acquired memories are lost, whereas remote memories, especially those acquired years before the damage, are more likely to be spared (Scoville and Milner 1957; Rempel-Clower et al. 1996). This pattern of amnesia is taken as evidence for temporally based systems consolidation, whereby over time the essential support for memories is "switched" from dependence on the HPC to neocortical networks (McClelland et al. 1995; Squire and Alvarez 1995; Anagnostaras et al. 2001; Meeter and Murre 2004; Squire et al. 2004; Wiltgen et al. 2004; Frankland and Bontempi 2005). Our research, however, points to another process for becoming independent of the HPC, a change in the strength of the representation in non-HPC systems during learning rather than a consolidation process linked to the passage of time since the learning episode. A study of a former London taxi driver with bilateral HPC damage alludes to this possibility (Maguire et al. 2006). This amnesic patient showed greater retrograde amnesia for roads that he used less commonly than the major arteries that he used regularly. Hence, greater exposure to the major arteries established memories in non-HPC systems, whereas roads with less exposure remained dependent on the HPC regardless of the age of the memory. Our findings add support to this view, because studies examining the effects of complete HPC damage after a single conditioning episode suggest that the HPC is permanently 
involved in contextual fear conditioning (Lehmann et al. 2007b; Sutherland et al. 2008); yet, with repeated learning episodes we clearly demonstrated that the memory rapidly becomes independent of the HPC. The latter is important because the process for memories becoming independent of the HPC need not require systems consolidation.

In conclusion, this is the first example of intact contextual fear memories following complete HPC damage induced soon after learning. Importantly, repetition of the learning episode underlies the change in memory from HPC dependent to HPC independent. We argue that each learning episode incrementally establishes a representation in non-HPC memory systems-a representation that ultimately becomes sufficiently strong to support memory expression without the HPC. The current findings also demonstrate the critical need to consider learning parameters when discussing patterns of retrograde amnesia and the role of the HPC in memory.

\section{Acknowledgments}

We acknowledge grant sponsors NIMH (MH61460), NSERC, AHFMR, and the Heart and Stroke Foundation of Canada.

\section{References}

Anagnostaras SG, Maren S, Fanselow MS. 1999. Temporally graded retrograde amnesia of contextual fear after hippocampal damage in rats: Within-subjects examination. J Neurosci 19: 1106-1114.

Anagnostaras SG, Gale GD, Fanselow MS. 2001. Hippocampus and contextual fear conditioning: Recent controversies and advances. Hippocampus 11: 8-17.

Antoniadis EA, McDonald RJ. 2000. Amygdala, hippocampus and discriminative fear conditioning to context. Behav Brain Res 108: $1-19$.

Biedenkapp JC, Rudy JW. 2009. Hippocampal and extrahippocampal systems compete for control of contextual fear: Role of ventra subiculum and amygdala. Learn Mem 16: 38-45.

Debiec J, LeDoux JE, Nader K. 2002. Cellular and systems reconsolidation in the hippocampus. Neuron 36: 527-538.

Driscoll I, Howard SR, Prusky GT, Rudy JW, Sutherland RJ. 2005. Seahorse wins all races: Hippocampus participates in both linear and nonlinear visual discrimination learning. Behav Brain Res 164: 29-35.

Fanselow MS, Poulos AM. 2004. The neuroscience of mammalian associative learning. Annu Rev Psychol 56: 207-234.

Frankland PW, Bontempi B. 2005. The organization of recent and remote memories. Nat Rev Neurosci 6: 119-130.

Frankland PW, Cestari V, Filipkowski RK, McDonald RJ, Silva AJ. 1998. The dorsal hippocampus is essential for context discrimination but not for contextual conditioning. Behav Neurosci 112: 863-874.

Guarraci FA, Frohardt RJ, Kapp BS. 1999. Amygdaloid D1 dopamine receptor involvement in Pavlovian fear conditioning. Brain Res 827: 28-40.

Kim JJ, Fanselow MS. 1992. Modality-specific retrograde amnesia of fear. Science 256: 675-677.

Lehmann H, Lecluse V, Houle A, Mumby DG. 2006. Retrograde amnesia following hippocampal lesions in the shock-probe conditioning test. Hippocampus 16: 379-387.
Lehmann H, Clark BJ, Whishaw IQ. 2007a. Similar development of cued and learned home bases in control and hippocampal-damaged rats in an open field exploratory task. Hippocampus 17: 370380.

Lehmann H, Lacanilao S, Sutherland RJ. 2007b. Complete or partial hippocampal damage produces equivalent retrograde amnesia for remote contextual fear memories. Eur J Neurosci 25: 1278-1286.

Maguire EA, Nannery R, Spiers HJ. 2006. Navigation around London by a taxi driver with bilateral hippocampal lesions. Brain 129: 2894-2907.

Maren S, Aharonov G, Fanselow MS. 1997. Neurotoxic lesions of the dorsal hippocampus and Pavlovian fear conditioning in rats. Behav Brain Res 88: $261-274$

McClelland JL, McNaughton BL, O'Reilly RC. 1995. Why there are complementary learning systems in the hippocampus and neocortex: Insights from the successes and failures of connectionist models of learning and memory. Psychol Rev 102: 419-457.

Meeter M, Murre JM. 2004. Consolidation of long-term memory: Evidence and alternatives. Psychol Bull 130: 843-857.

Moscovitch M, Nadel L, Winocur G, Gilboa A, Rosenbaum RS. 2006. The cognitive neuroscience of remote episodic, semantic and spatial memory. Curr Opin Neurobiol 16: 179-190.

O'Keefe JM, Nadel L. 1978. The hippocampus as a cognitive map. Oxford University Press, Oxford, UK.

O'Reilly RC, Rudy JW. 2001. Conjunctive representations in learning and memory: Principles of cortical and hippocampal function. Psychol Rev 108: $311-345$.

Rempel-Clower NL, Zola SM, Squire LR, Amaral DG. 1996. Three cases of enduring memory impairment after bilateral damage limited to the hippocampal formation. J Neurosci 16: 5233-5255.

Scoville WB, Milner B. 1957. Loss of recent memory after bilateral hippocampal lesions. J Neurochem 20: 11-21.

Sherry DF, Schacter DL. 1987. The evolution of multiple memory systems. Psychol Rev 94: 439-454.

Squire LR, Alvarez P. 1995. Retrograde amnesia and memory consolidation: A neurobiological perspective. Curr Opin Neurobiol 5: 169-177.

Squire LR, Stark CE, Clark RE. 2004. The medial temporal lobe. Annu Rev Neurosci 27: 279-306.

Stout S, Arcediano F, Escobar M, Miller RR. 2003. Overshadowing as a function of trial number: Dynamics of first- and second-order comparator effects. Learn Behav 31: 85-97.

Sutherland RJ, Lehmann H, Spanswick SC, Sparks FT, Melvin NR. 2006. Growth points in research on memory and hippocampus. Can J Exp Psychol 60: $166-174$.

Sutherland RJ, O'Brien J, Lehmann H. 2008. Absence of systems consolidation of fear memories after dorsal, ventral, or complete hippocampal damage. Hippocampus 18: 710-718.

Wang SH, Teixeira CM, Wheeler AL, Frankland PW. 2009. The precision of remote context memories does not require the hippocampus. Nat Neurosci 12: 253-255.

White NM, McDonald RJ. 2002. Multiple parallel memory systems in the brain of the rat. Neurobiol Learn Mem 77: 125-184.

Wiltgen BJ, Brown RA, Talton LE, Silva AJ. 2004. New circuits for old memories: The role of the neocortex in consolidation. Neuron 44: 101-108.

Wiltgen BJ, Sanders MJ, Anagnostaras SG, Sage JR, Fanselow MS. 2006. Context fear learning in the absence of the hippocampus. J Neurosci 26: 5484-5491.

Winocur G, Moscovitch M, Sekeres M. 2007. Memory consolidation or transformation: Context manipulation and hippocampal representations of memory. Nat Neurosci 10: 555-557.

Received February 24, 2009; accepted in revised form April 27, 2009. 


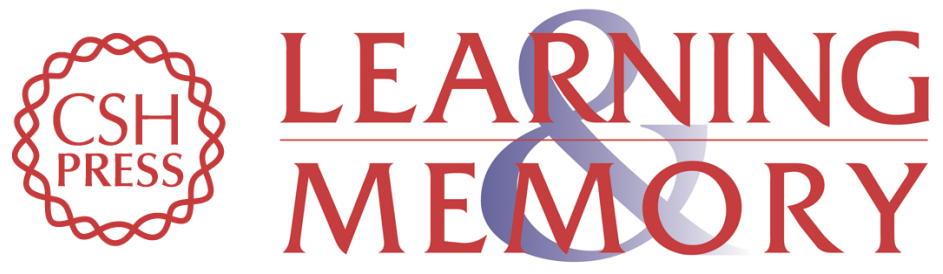

\section{Making context memories independent of the hippocampus}

Hugo Lehmann, Fraser T. Sparks, Simon C. Spanswick, et al.

Learn. Mem. 2009, 16:

Access the most recent version at doi:10.1101/lm.1385409

Supplemental http://learnmem.cshlp.org/content/suppl/2009/06/29/16.7.417.DC1

Material

References This article cites 33 articles, 4 of which can be accessed free at: http://learnmem.cshlp.org/content/16/7/417.full.html\#ref-list-1

License

Email Alerting Receive free email alerts when new articles cite this article - sign up in the box at the Service top right corner of the article or click here. 